\title{
Laser detection using liquid crystal polarization modulators
}

\author{
David M. Benton* \\ Aston University, Aston Institute of Photonic Technologies, Birmingham, United Kingdom
}

\begin{abstract}
Lasers can be identified by their relatively long coherence lengths using interferometry. A Mach-Zehnder interferometer incorporating liquid crystal polarization modulators is demonstrated as a means of low-cost, robust laser detection. Temporal modulations, as a signature of coherence, can be induced by modulating polarization changes in liquid crystal modulators using low voltages. Sensitivities of $<10 \mathrm{nW}$ can be achieved. The suitability as a means of laser detection is discussed. (C) 2020 Society of Photo-Optical Instrumentation Engineers (SPIE) [DOI: 10.1117/1.OE.59.6.064106]
\end{abstract}

Keywords: laser detection; interferometry; liquid crystal.

Paper 20200378 received Apr. 6, 2020; accepted for publication Jun. 1, 2020; published online Jun. 8, 2020.

\section{Introduction}

Lasers are now a pervasive technology with many familiar applications that range from communication, material processing, three-dimensional scanning, printing, medical applications, and many more. The generation of laser radiation requires such an imbalance of thermodynamic equilibrium that only highly unusual astrophysical scale phenomena can generate lasing in nature. ${ }^{1}$ All other lasers are engineered and used with a purpose. It is the intent behind the use that drives military desire to detect lasers. Military applications of lasers include range finding, target designation, laser dazzle, and missile control. ${ }^{2}$ The majority of lasers of interest to the military are pulsed lasers. The temporal resolution and high instantaneous brightness make them well-suited for operating over ranges of many kilometers in applications such as range finders and target markers. Generations of laser Warner receivers (LWRs) have been developed to detect the threat posed by these lasers ${ }^{3,4}$ and allow irradiated platforms to initiate appropriate countermeasures determined by the perceived threat. In the last few years, a more widespread hazard has arisen from the preponderance of continuous wave $(\mathrm{CW})$ visible laser diodes: so-called laser pointers. Handheld visible lasers with powers of several Watts are available for a few hundred dollars with an ever-expanding range of wavelengths. These lasers have proven to be a menace when directed toward aircraft as they approach landing, with over 1500 reports of lasers dazzling pilots in the United Kingdom and United States last year. Conventional LWRs do not perform well at detecting these lasers because they rely on the rapid temporal changes in brightness observed with pulsed lasers. CW lasers are therefore a specific challenge in the world of laser detection.

Wang et al..$^{5}$ divided laser detection into three categories: coherent recognition, scattering recognition, and spectrum recognition. These are categories based on what is observed rather than discriminating characteristic. Benton et al. ${ }^{6}$ took a classification approach based on the discrimination technique, classifying them into imaging, spectral, or coherence. The first two categories are both essentially discriminating based on brightness. Imaging systems make use of CCD arrays ${ }^{7,8}$ and intensified cameras, ${ }^{2}$ while semi-imaging systems look for tell-tale circles from bright sources. ${ }^{9}$ Spectroscopic systems typically use dispersion with a diffraction grating and a detector array. ${ }^{10,11}$ Discrimination based on coherence detection is the main subject of this paper.

The concept of using the coherence properties of laser radiation as its signature of detection has been around for decades with the underlying principle being that broadband background

*Address all correspondence to David M. Benton, E-mail: d.benton@aston.ac.uk 
radiation has a very short coherence length (typically microns) that suppresses interference effects in interferometers that are unbalanced (asymmetric). Fabry-Perot interferometers with angle scanning have been used by $\mathrm{Crane}^{12,13}$ for detecting pulsed and $\mathrm{CW}$ lasers. Michelson interferometers have been used as coherence detectors by which long path length differences are able to determine the coherence length of the source ${ }^{14,15}$ When used with an imaging system, this is able to remove background illumination. ${ }^{16}$ Multiple interferometer paths are able to act as a coherence length bandpass filter. ${ }^{17}$

The Fizeau interferometer has been also been considered in the literature. ${ }^{10,18}$ Benton ${ }^{19}$ made use of a Mach-Zehnder interferometer (MZI) to produce a low-cost laser detection system capable of discriminating wavelength. Cost is an important matter as typically interferometric detection systems can be fragile and, consequentially, heavy and expensive. This provides an economic asymmetry in which the detectors for lasers are significantly more expensive than the laser threats that they are detecting. Cost effective detectors will rebalance this asymmetry to some extent.

The requirement for robust detectors suggests that any system using a moving mirror may be overly fragile, and thus a system with no "moving parts" would be desirable. To meet this requirement, an interferometric system based on polarization modulation has been investigated. Such a system has been investigated by Cohen, ${ }^{20}$ but the use of electrooptic modulators, which are (at that time) bulky, require high voltage, and are expensive, does not meet the desire for lowcost devices. In this paper, polarization modulation is achieved through the use of liquid crystal modulators (LCM), which are compact, require low voltage, and are relatively cheap to produce.

Unlike the system of Cohen,${ }^{20}$ the LCMs are incorporated into an MZI, building on previous work ${ }^{19}$ as a method of obtaining a low-cost laser detection system.

\section{Methods}

A generic MZI with a polarization modulator is shown generically in Fig. 1. Introducing a polarization-dependent component into an MZI causes two orthogonal polarizations to be present with independent interference regimes, but these are not distinguished by the detectors. Any interference seen by the detector is the net result of the two orthogonal polarizations detected simultaneously. (Note that using polarization-sensitive beam splitters does not work here.) Changing the applied voltage to the LCM modulates the refractive index of one polarization axis. Thus, the time-dependent intensity varies for only one polarization component. Laser sources are, in general, polarized, and this will be at some unknown angle. By considering the simple but instructive case of linear input polarization, we can model the expected system behavior.

The intensity in detectors 1 and 2 in each polarization mode (labeled $x$ and $y$ ) are given from the standard MZI interference equation ${ }^{19,20}$ as

$$
\begin{aligned}
& I_{1, x}=2 I_{0} \sin (k \theta)^{2} B^{2}\left\{1+\cos \left[\pi \frac{v(t)}{V_{\pi}}\right]\right\}, \\
& I_{1, y}=2 I_{0} \cos (k \theta)^{2} B^{2}, \\
& I_{2, x}=2 I_{0} \sin (k \theta)^{2} B^{2}\left\{1-\cos \left[\pi \frac{v(t)}{V_{\pi}}\right]\right\}, \\
& I_{2, y}=2 I_{0} \cos (k \theta)^{2} B^{2},
\end{aligned}
$$

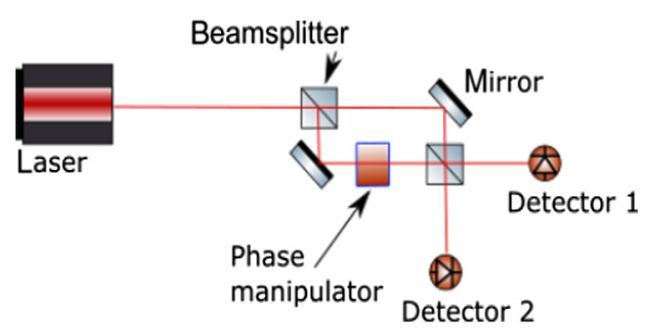

Fig. 1 A generic MZl. 
where $k=2 \pi / \lambda$, with $\lambda$ being the wavelength, $\theta$ is the polarization angle of the incoming light relative to the $x$ axis, $B$ is the beam splitting ratio assuming equal reflection and transmission $(B=0.5), v(t)$ is the time varying voltage applied to the electrodes of the LCM, and $V_{\pi}$ is the voltage required to generate a phase shift of $\pi$. Plots of the normalized intensity level at one of the detectors can be produced as the input polarization is varied. The plots show the intensity at the minimum voltage level $V_{0}$ and the maximum voltage level $V_{0}+V_{\pi}$. In each case, the modulation amplitude is chosen to be $V_{\pi}$ as this gives the maximum modulation response in the detectors (although it is wavelength dependent). These plots can be seen in Fig. 2. The top left plot shows that there are polarization values where the intensity at the max and min values is the same, and no modulation signal will be observed. This is because when the input polarization is orthogonal to the LCM modulation axis there is no effect. To overcome this, consider the case of 2 such LCMs, one in each arm, oriented orthogonally to each other:

$$
\begin{aligned}
& I_{1, x}=2 I_{0} \sin (k \theta)^{2} B^{2}\left\{1+\cos \left[\pi \frac{v_{a}(t)}{V_{\pi}}\right]\right\}, \\
& I_{1, y}=2 I_{0} \cos (k \theta)^{2} B^{2}\left\{1+\cos \left[\pi \frac{v_{b}(t)}{V_{\pi}}\right]\right\}, \\
& I_{2, x}=2 I_{0} \sin (k \theta)^{2} B^{2}\left\{1-\cos \left[\pi \frac{v_{a}(t)}{V_{\pi}}\right]\right\}, \\
& I_{2, y}=2 I_{0} \cos (k \theta)^{2} B^{2}\left\{1-\cos \left[\pi \frac{v_{b}(t)}{V_{\pi}}\right]\right\} .
\end{aligned}
$$

Here, we label the voltages with the subscripts $a$ and $b$ to represent the modulation voltages applied to each modulator. Applying the same signal to both modulators (oriented orthogonally), we get the result for min and max intensity values as shown in the top right of Fig. 2. Here we can see that there is a modulation signal to be observed at all polarizations. By adding a relative phase
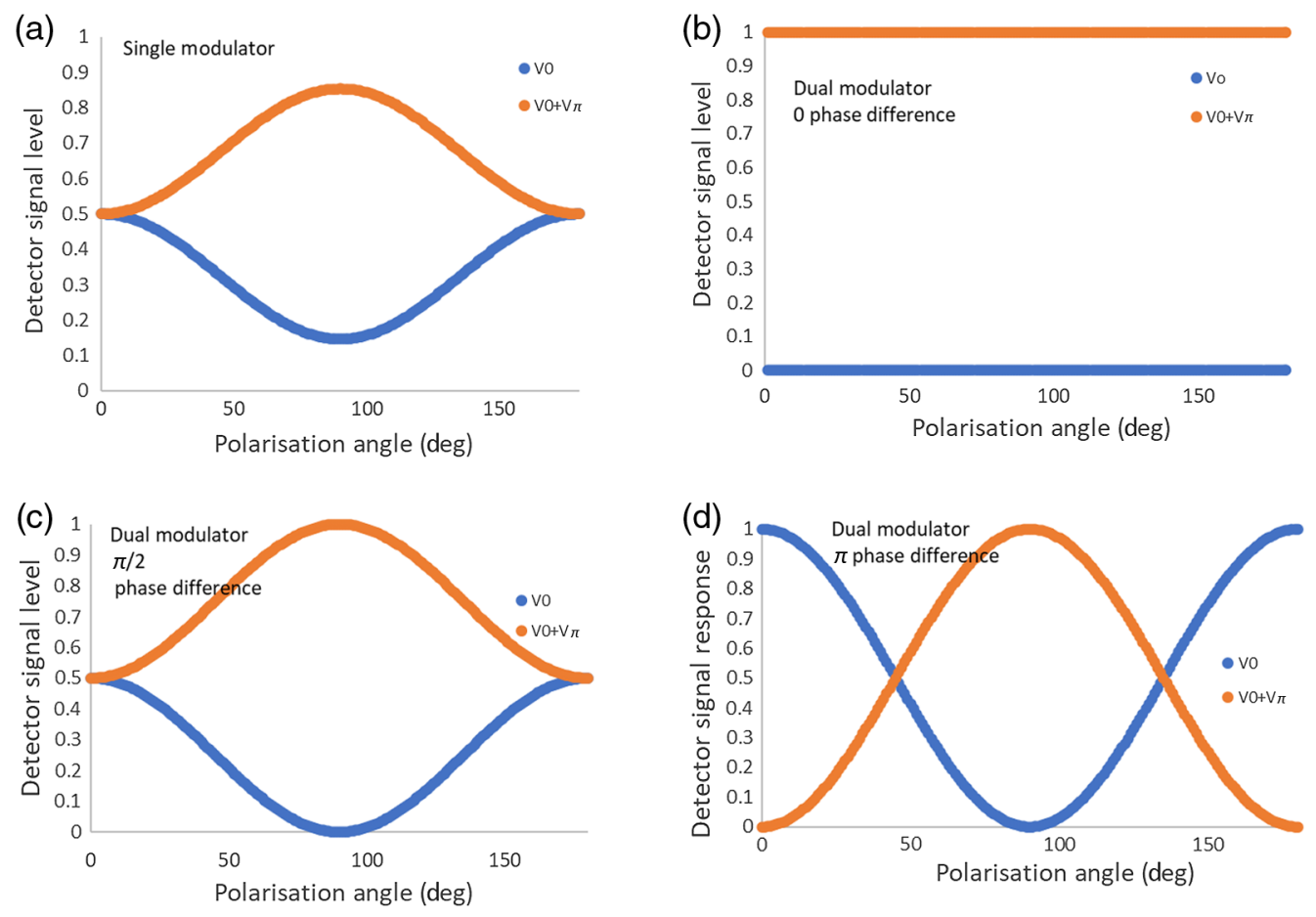

Fig. 2 Plots of modeling signal intensity at one of the detectors as input polarization is varied. All plots show the normalized intensity at the $\min \left(V_{0}\right)$ and $\max \left(V_{0}+V_{\pi}\right)$ voltages. (a) A single modulator. (b) Two orthogonal modulators with no phase difference between signals. (c) $A \pi / 2$ phase difference. (d) A $\pi$ phase difference. 


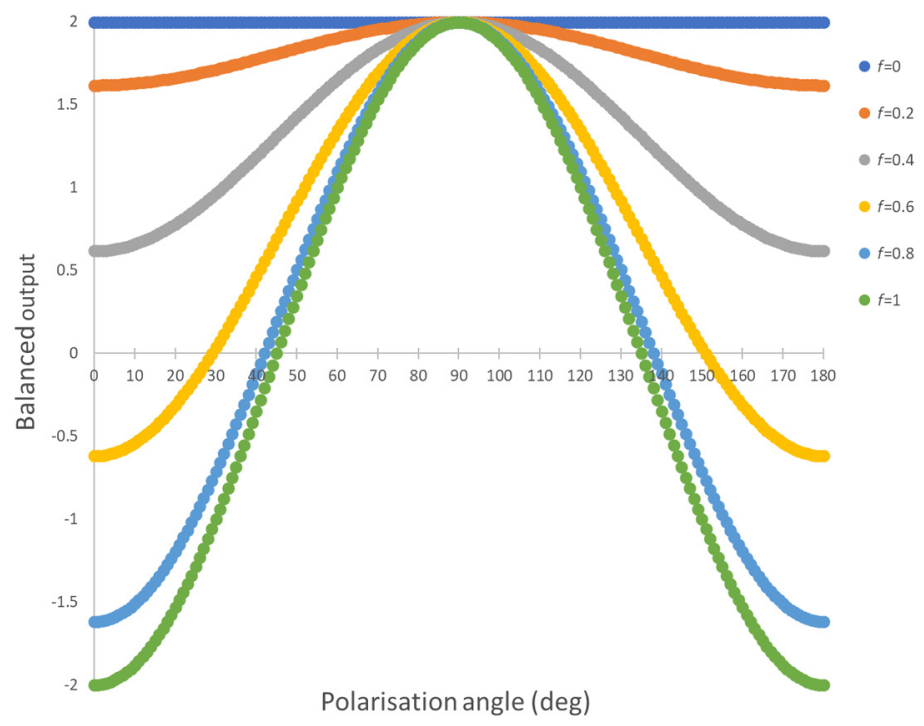

Fig. 3 Balanced detector relative signals vs polarization angle for differing phase offset between the 2 modulators-given as a fraction of pi.

difference to the modulators, we can change the behavior as shown with a $\pi / 2$ phase difference (bottom right) and a $\pi$ phase difference (bottom left). The amplitude of the modulating signal in the detector is the difference between the values for the max and min applied voltages. To represent the signal size obtained from a balanced system, we must subtract signals from the two detectors, which are in antiphase. Figure 3 shows plots of the system signal strength for differing polarization values where the phase difference between the signals to each modulator is given as a fraction, $f$, of $\pi$. It would appear that a system with no phase difference between (orthogonally oriented) modulators would be most reliable, but it is possible that the behavior seen with some phase difference could be useful in helping to determine the incoming wavelength, such as by observing the difference in signal as the phase is changed.

\section{Experimental Setup}

An MZI incorporating LCMs is shown schematically in Fig. 4. This system is composed of two nonpolarizing beam splitters dividing light toward two adjustable mirrors. Two polarization modulators (Thorlabs LCC1111U-A) were located, one in each path. The electrical signals from two silicon photodiodes were amplified and sent to a data acquisition unit (National Instruments USB 6341) and then to a computer where the signals were processed using a LabView program.

Applying a voltage across the LCM requires the regular polarity reversal of the voltage across the device electrodes to prevent the permanent displacement of the LC medium causing performance reduction. This polarity reversal is usually done at a frequency of a few $\mathrm{kHz}$.

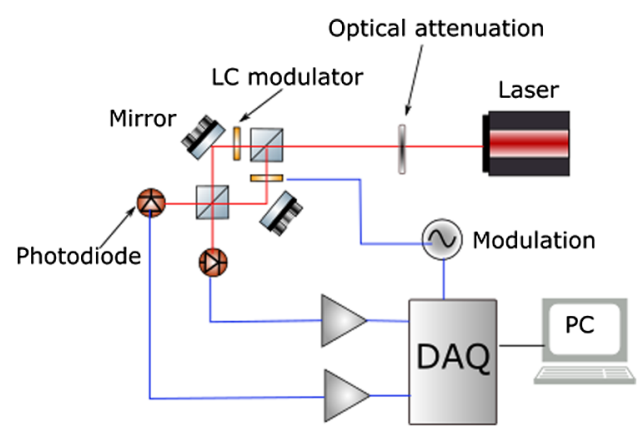

Fig. 4 A schematic diagram of the laser detection system with polarization modulators in an MZI. 
Modulating the device voltage to change polarization properties involves changing the amplitude of this "carrier" frequency. A modulation waveform was generated using the DAQ device with two analog-to-digital converters connected to the LCM electrodes. The amplitude, carrier frequency, and modulation frequency were controlled using a LabView program.

\section{Results}

It is first necessary to characterize the response of the LCM. This was done using a polarizing beamsplitter with two photodiodes measuring the intensity of each output polarization and an input laser polarized at $45 \mathrm{deg}$. A modulation frequency was applied to the LCM, and the resulting intensities at the detectors were subtracted to produce a measure of the amplitude of the polarization effect being induced. The laser wavelength being used was $635 \mathrm{~nm}$ from a laser diode, and the amplitude of the applied voltage was $\pm 2.5 \mathrm{~V}$, which was found to produce a significant level of response for this wavelength. A plot of the polarization response versus modulation frequency is shown in Fig. 5. This clearly shows that the amount of polarization response drops rapidly as the frequency is increased. The trendline is a fourth power polynomial. Thus, it can be seen that it is preferential to operate these devices at relatively low frequencies of a few 10 s of $\mathrm{Hz}$.

Using the MZI setup as shown in Fig. 6, the interferogram in Fig. 4. shows the signal in both detectors for an applied modulation frequency of $32 \mathrm{~Hz}$ with a sampling rate of $20 \mathrm{kHz}$ and an input laser at $630 \mathrm{~nm}$. This clearly shows the modulation of the signal in both detectors changing in antiphase. As was the case in Ref. 19, the strength of the received signal at the modulation frequency is measured by taking the Fourier transform of the interferogram and determining the power at the modulation frequency.

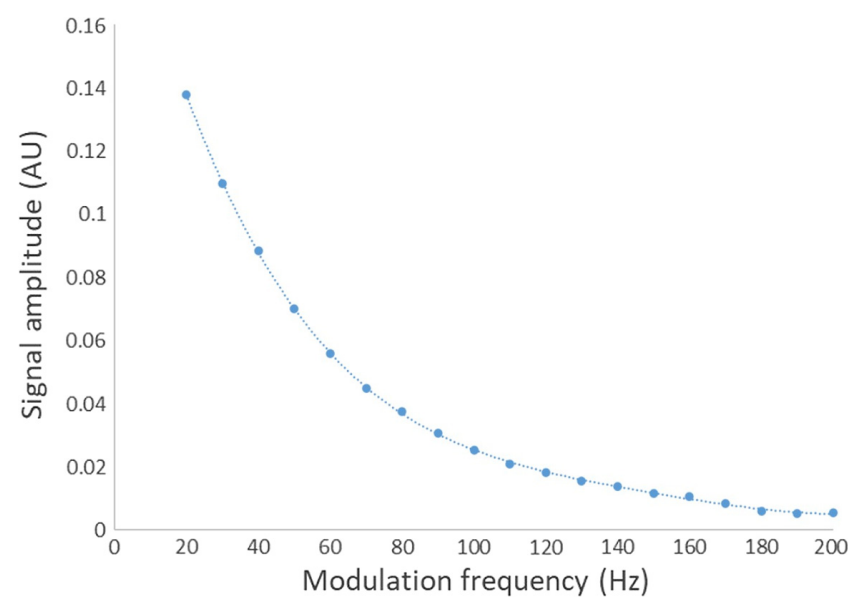

Fig. 5 A plot of the polarization effect produced by the LCMs as frequency is varied.

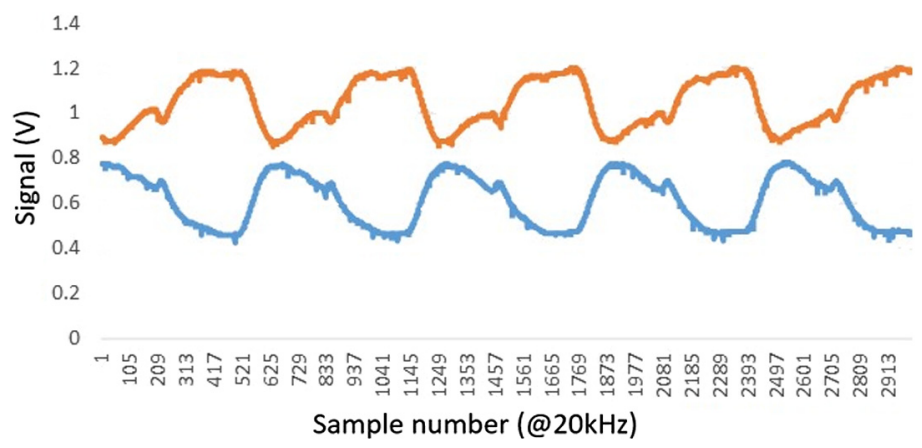

Fig. 6 Modulated intensity seen in both detectors as a modulating voltage is applied to an LCM. 


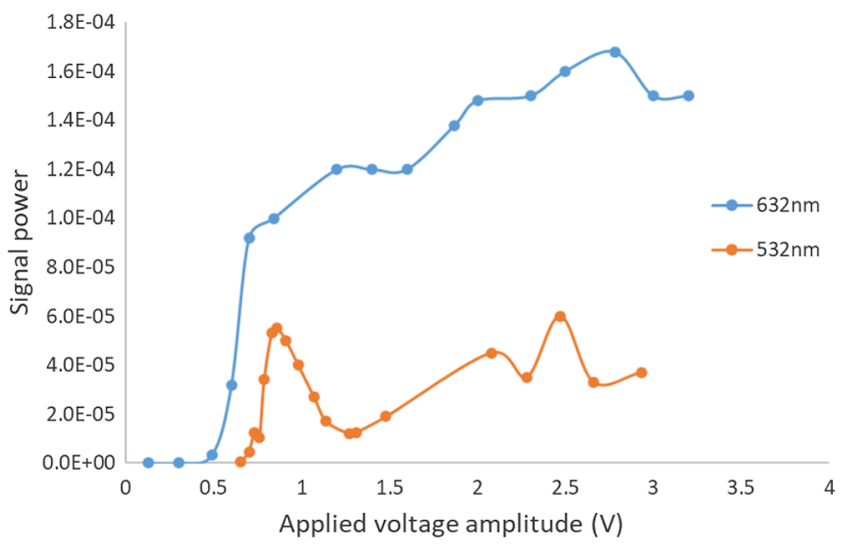

Fig. 7 Signal power at the modulation frequency for varying amplitudes of drive modulation.

Varying the amplitude of the modulation alters the amount of path length difference for one polarization, an effect which is wavelength dependent. Figure 7 plots the detected signal power against the amplitude of the modulation applied (at $20 \mathrm{~Hz}$ ) to the LCM. This is shown for two wavelengths: 632 and $532 \mathrm{~nm}$. This was also attempted at $405 \mathrm{~nm}$, but no modulation could be observed, despite the device specifications claiming operation out to $350 \mathrm{~nm}$. An amplitude of around $\pm 2.5 \mathrm{~V}$ is a useful level that gives good response.

The effect of the polarization angle of the input laser was examined and can be seen in Fig. 8. The system was driven at a frequency of $20 \mathrm{~Hz}$ and with an amplitude of $2.5 \mathrm{~V}$. The DC level of the detected signal is shown representing the laser power, and it shows that there is only a small variation of power with polarization angle. With a single modulator operating, there were found to be two positions with maximum response and two with very low response, corresponding to polarization alignment with the LCM axes.

Clearly this means that there are situations in which even an intense laser would generate no modulation power at certain polarization angles. Such a device would have only limited utility. The process was repeated with a second LCM oriented orthogonally to the first but driven with the same modulation. This shows that modulation power is observed at all input polarization angles.

Using two LCMs presents us with the possibility of controlling the devices independently. In the present case, this caused a problem as the DAQ device has only two analog outputs. However, the convenient choice of $\pm 2.5 \mathrm{~V}$ applied to each electrode is equivalent to switching

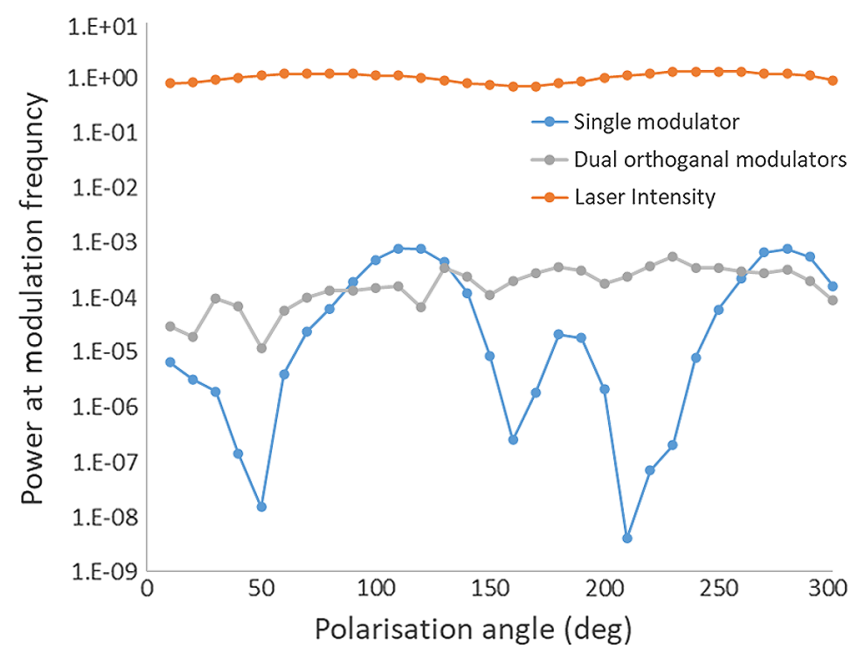

Fig. 8 The variation of MZI system response to changing input polarization. Data shows the laser intensity and the effect for a single modulator and a dual modulator system. 


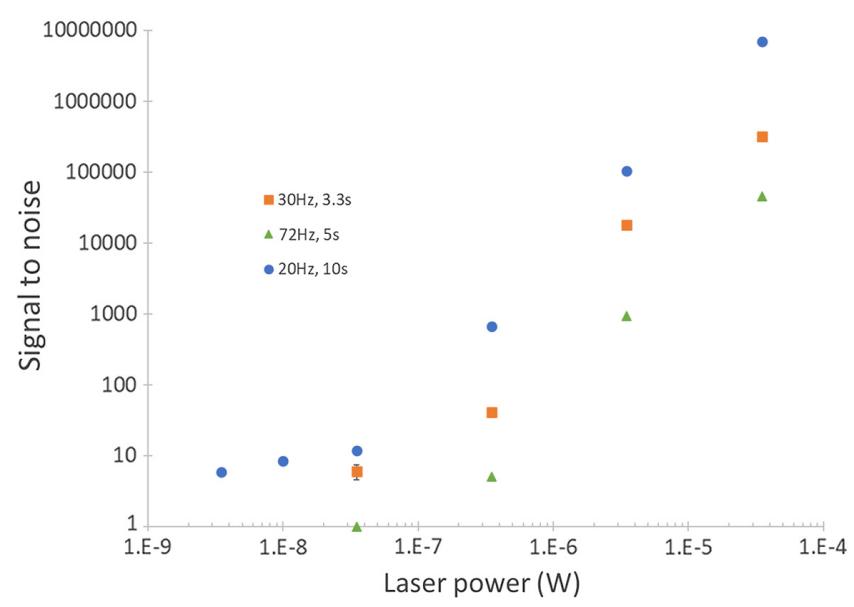

Fig. 9 Plots of the signal-to-noise for different modulation drive frequencies and differing signal collection times.

between 0 and $5 \mathrm{~V}$, and hence four digital output lines could be used. While not allowing independent control of amplitude, the LCMs can be modulated at different frequencies; this could enable, for example, looking for the resultant modulation power at the sum frequency, which may have advantages such as better noise. The digital system switched at a carrier frequency of $2 \mathrm{kHz}$ with the modulation frequency applied by shifting the phase of the two electrodes by $\pi$ every half period. Two independent frequencies were observed in the detector output, but at this point it was clear that one of the LCMs had significantly degraded (it was older and had more use) and produced less modulation power. Also the net effect was to reduce the modulation power relative to both LCMs having the same frequency. While no significant results can be presented for this, it is included for completeness.

The detection sensitivity of the system was examined using the digital modulation with both LCMs present. This was conducted with a diode laser at $635 \mathrm{~nm}$ and various levels of attenuation using neutral density filters. Consecutive Fourier transform power spectra were summed to increase sensitivity by increasing integration time. Various modulation frequencies and integration times were examined. Results of the signal to noise levels recorded are shown in Fig. 9. As expected, sensitivity at the highest frequency examined $(72 \mathrm{~Hz})$ is worst with a sensitivity limit $(S: N=1)$ of around $20 \mathrm{nW}$ (5s integration). The best response for a $20-\mathrm{Hz}$ modulation signal with a 10-s integration time suggests that a sensitivity of around $1 \mathrm{nW}$ is possible. Normalizing the responses to a 1-s integration time suggests a detection threshold of $10 \mathrm{nW}$ at $20 \mathrm{~Hz}$ and $60 \mathrm{nW}$ at $72 \mathrm{~Hz}$.

\section{Conclusions}

The motivation behind this work was to continue the development of low-cost laser detection based upon coherence detection as given in Ref. 19. The use of LCMs replaces the piezo modulated mirror as it is always desirable to have no moving parts, particularly those with critical alignment. The LCMs have been successfully used within an asymmetric MZI as a means of modulating coherent light, and with no moving parts, they certainly lead to a more robust system that is easier to align and maintain. The use of two LCMs oriented orthogonally has been used to overcome the limitation of response to particular polarization angles seen with a single modulator. The LCMs require only low voltage signals, which is an advantage over, for example, electro-optic polarization modulators, and have been used with a digital drive signal. Their use has been targeted at CW lasers, in particular the laser pointer type. These lasers may typically have short coherence lengths $(<1 \mathrm{~cm}$, sometimes $<1 \mathrm{~mm})$. The asymmetry of the MZI arms needs only to be a few microns of length difference to prevent background contributions, which is easily achievable. Thus, this is a viable technology for laser pointer detection. 
However, there is no hiding the limitations that arise from the LCMs themselves. Most notable is the limitation of the inherent switching frequency of the devices. The LCM response is best at low frequencies around a few tens of $\mathrm{Hz}$, which naturally limits the speed and sensitivity of response of the system. In critical situations, this slow response would be unacceptable. Also, the effect of atmospheric scintillation leading to intensity modulations upon a transiting laser is significant at these low frequencies, especially in comparison with devices that operate beyond $1 \mathrm{kHz}$. The LCMs chosen were designed for use in the visible part of the spectrum. LCMs are available for the near-IR, but these devices have a thicker layer of liquid crystal and have a slower response time. However, the slow modulation rates of LCMs are very well suited to use with cameras instead of photodiodes. These modulation rates can show up as temporal modulations at specific spatial locations, defining the origin of the laser source (with suitable video processing). This may be a more promising line of future investigation. As with most technologies, there are particular situations for which they are well suited. These LCM-based interferometers have a slower response and are less sensitive, which limits their applicability, but they may be relevant when faster LCMs or alternative devices are available.

\section{References}

1. S. Chen, "Alien light," https://spie.org/news/photonics-focus/janfeb-2020/astrophysicallasers?SSO=1 (2020).

2. J. Dubois and F. Reid, "Detecting laser sources on the battlefield," Proc. SPIE 6796, 67962F (2007).

3. J. Pietrzak, "Laser warning receivers," Proc. SPIE 5229(2), 318-322 (2003).

4. M. Dąbrowski et al., "Laser Warner receiver LWR-H," Proc. SPIE 6598, 65980S (2006).

5. L. Wang et al., "Optimum design of wideangle laser detecting system based on fisheye lens and sinusoidal amplitude grating," Opt. Commun. 310, 173-178 (2014).

6. D. M. Benton, M. A. Zandi, and K. Sugden, "Laser detection utilizing coherence," Proc. SPIE 11161, 111610G (2019).

7. S. Kumar et al., "Design of a laser-warning system using an array of discrete photodiodes: part I," J. Battlefield Technol. 14(1), 13 (2011).

8. J. Ying and Z. Zhou "Study on image processing technology in imaging laser detection system," in Symp. Photonics and Optoelectron., IEEE (2010).

9. S. Tipper, C. Burgess, and C. Westgate, "Novel low-cost camera-based continuous wave laser detection," Proc. SPIE 11019, 110190B (2019).

10. A. D. McAulay, "Detecting modulated lasers in the battlefield and determining their direction," Proc. SPIE 7336, 73361J (2009).

11. J. Zhang, E. Tian, and Z. Wang, "Research on laser warning receiver based on sinusoidal transmission grating and high speed DSPs," WSEAS Trans. Circuits Syst. 5(8), 1366-1371. (2006).

12. R. Crane, Jr., "Laser detection by coherence discrimination," Opt. Eng. 18(2), 182212 (1979).

13. R. Crane, Jr., “The angle-scanned interferometer," Opt. Eng. 18(2), 182205 (1979).

14. D. Hickman, "An optical sensor based on temporal coherence properties," J. Sci. Instrum. 21, 187-192 (1988).

15. R. C. Coutinho et al., "Detection of coherent light in an incoherent background [for IRST]," in 1999 IEEE LEOS Annu. Meeting Conf. Proc. LEOS'99. 12th Annu. Meeting, IEEE Lasers and Electro-Optics Society 1999 Annu. Meeting (Cat. No. 99CH37009), IEEE, Vol. 1, pp. 247-248 (1999).

16. C. J. Duffey and D. Hickman, "An imaging system based on temporal coherence differences," J. Phys. D: Appl. Phys. 21, S56-S58 (1988).

17. P. Sutton, "A novel electro-optical remote-sensing technique based on bandpass coherence processing," J. Phys. 22, 379 (1989).

18. J. C. Russell, "Coherent laser warning system," Patent No. 6,151,114 (2000).

19. D. M. Benton, "Low-cost detection of lasers," Opt. Eng. 56(11), 114104 (2017).

20. J. D. Cohen, "Electrooptic detector of temporally coherent radiation," Appl. Opt. 30(7), 874-883 (1991). 
David M. Benton graduated in physics from the University of Birmingham in 1989. He received his PhD in laser spectroscopy for nuclear physics in 1994 and then conducted postdoctoral research in positron emission tomography and then laser spectroscopy for nuclear physics, all at the University of Birmingham. In 1998, he joined DERA, which became QinetiQ, where he worked on a variety of optical projects. He was the leader of a group building quantum cryptography systems and was involved in a notable $140 \mathrm{~km}$ demonstration in the Canary Islands. He became chief scientist for L-3 TRL in 2010, working on photonic processing techniques for RF applications. He is now at Aston University with a variety of research interests including encoding techniques, gas sensing, and laser detection techniques. He is a member of SPIE. 